\title{
ON THE EVALUATION MAP
}

\author{
ANICETO MURILLO
}

\begin{abstract}
The evaluation map of a differential graded algebra or of a space is described under two different approaches. This concept turns out to have geometric implications: (i) A 1-connected topological space, with finite-dimensional rational homotopy, has finite-dimensional rational cohomology if and only if it has nontrivial evaluation map. (ii) Let $E \stackrel{\rho}{\rightarrow} B$ be a fibration of simplyconnected spaces. If the rational cohomology of the fibre is finite dimensional and the evaluation map of the base is different from zero, then the evaluation map of the total space is nonzero. Also, if $\rho$ is surjective in rational homotopy and the evaluation map of $E$ is nontrivial, then the evaluation map of the fibre is different from zero.
\end{abstract}

\section{INTRODUCTION}

The evaluation map of an augmented differential graded algebra (DGA) $A$ was first defined in [3] as a natural vector map,

$$
\mathrm{ev}_{A}: \operatorname{Ext}_{A}(\mathbb{K}, A) \rightarrow H(A) .
$$

The evaluation map $\mathrm{ev}_{S}$, of a pointed topological space is, by definition, the evaluation map of the DGA $C^{*}(S ; \mathbb{K})$. If $\mathrm{ev}_{S} \neq 0$ there are interesting topological consequences for $S$. For instance it follows [3] that

$$
\operatorname{Ext}_{H_{*}(\Omega S ; \mathbf{K})}\left(\mathbb{K},\left(H_{*}(\Omega S ; \mathbb{K})\right)\right) \neq 0
$$

and this leads to important structural constraints on the Hopf algebra $H_{*}(\Omega S ; \mathbb{K})$ (see for example [2]). On the other hand, simple topological conditions can force $\mathrm{ev}_{S}$ to be nonzero. For instance, $\mathrm{ev}_{S}$ is nonzero [3, Proposition 1.6] if $S$ has the form $T \cup_{f} e^{n+1}$ with the characteristic class of $e^{n+1}$ nonzero in $H^{n+1}(S ; \mathbb{K})$. In this case we say $S$ has a terminal cell.

In this paper we find other natural geometric conditions which imply that the evaluation map is nonzero, and give further geometric consequences of this nonvanishing.

Our first goal is a characterization of 1-connected rationally elliptic spaces in terms of their evaluation map. A 1-connected space $S$ is rationally elliptic if the vector spaces $\pi_{*}(S) \otimes \mathbb{Q}$ and $H^{*}(S ; \mathbb{Q})$, are finite-dimensional [1]. Homogeneous spaces are classical examples of such spaces. We prove

Received by the editors February 2, 1990 and, in revised form, June 14, 1991.

1980 Mathematics Subject Classification (1985 Revision). Primary 55P62.

Key words and phrases. Evaluation map, rational homotopy, Sullivan models, elliptic spaces.

Partially supported by a DGICYT grant (PB88-0329). 
Theorem A. Let $S$ be a 1-connected pointed space with $\pi_{*}(S) \otimes \mathbb{Q}$ finite-dimensional. Then the following statements are equivalent:

(i) $H^{*}(S ; \mathbb{Q})$ is finite-dimensional.

(ii) $\operatorname{ev}_{S}($ over $\mathbb{Q})$ is different from zero.

Observe that the finiteness of the dimension of $\pi_{*}(S) \otimes \mathbb{Q}$ is necessary as is shown in the following:

Example. The space $\mathbb{C} P^{\infty} \vee S^{n}$ has infinite-dimensional rational cohomology and its evaluation map is different from zero, since it has a terminal cell.

Theorem $\mathrm{A}$ is a first approximation to the main result of this paper:

Theorem B. Let $F \rightarrow E \stackrel{\rho}{\rightarrow} B$ be a fibration of simply-connected spaces.

(i) If $H^{*}(F ; \mathbb{Q})$ is finite-dimensional, then $\mathrm{ev}_{B} \neq 0$ implies $\mathrm{ev}_{E} \neq 0$.

(ii) if $\pi_{*}(F) \otimes \mathbb{Q}$ is finite-dimensional and $\pi_{*}(\rho) \otimes \mathbb{Q}$ is surjective, then $\mathrm{ev}_{E} \neq 0$ implies $\mathrm{ev}_{F} \neq 0$.

In particular, in view of Theorem $\mathrm{A}$, we deduce the following:

Corollary. Given a fibration $F \rightarrow E \stackrel{\rho}{\rightarrow} B$ of simply-connected spaces in which $\pi_{*}(F) \otimes \mathbb{Q}$ is finite-dimensional and $\pi_{*}(\rho) \otimes \mathbb{Q}$ is surjective, $\mathrm{ev}_{E} \neq 0$ implies $\operatorname{dim} H^{*}(F ; \mathbb{Q})<\infty$.

Remark. As we observed above the hypothesis $\mathrm{ev}_{E} \neq 0$ holds, for example, if $E$ has a terminal cell.

In the next section we recall some notions and basic facts in Sullivan's theory of minimal models, its connection with rational homotopy theory, and differential homological algebra. In $\S 2$ we give two definitions of the evaluation map and establish their equivalence. In $\S 3$ we prove the algebraic results from which Theorem B(i) and Theorem A are deduced. Finally, in $\S 4$, we shall prove Theorem B(ii) from its "algebraic translation".

\section{BASIC FACTS}

All prerequisites in Sullivan's theory of minimal models and its connection with rational homotopy theory can be found in $[1,5,10]$. We shall follow the notation in [1 and 5].

All graded vector spaces are defined over a field $\mathbb{K}$ of characteristic zero unless stated otherwise. The differential of a DGA (differential graded algebra) raises the degree by one. Unadorned tensor products are tensor products over $\mathbb{K}$. In a graded object, degrees are superscript and we shall denote the degree of an element $x$ by $|x|$.

Given two differential graded vector spaces $V$ and $W$, their complete tensor product $V \hat{\otimes} W$ is another differential graded vector space defined as follows:

$(V \hat{\otimes} W)^{p}=\Pi_{i}\left(V^{i} \otimes W^{p-i}\right), \quad(d \Phi)_{i}=d_{V} \Phi_{i-1}+d_{W} \Phi_{i}, \quad \Phi=\left\{\Phi_{i}\right\} \in(V \hat{\otimes} W)^{p}$.

Observe that an element in $(V \hat{\otimes} W)^{p}$ can be written as $\sum_{i \in \mathbf{Z}} v_{i} \otimes w_{i}$, where $\left|v_{i}\right|+\left|w_{i}\right|=p,\left|v_{i}\right| \leq\left|v_{i+1}\right|$, and for each $q \in \mathbb{Z}$ the set $\left\{v_{i},\left|v_{i}\right|=q\right\}$ is finite. The semicomplete tensor product $V \tilde{\otimes} W$ is the subspace of $V \hat{\otimes} W$ formed by the elements $\sum_{i \geq 0} v_{i} \otimes w_{i}$, i.e., $V \tilde{\otimes} W=\underline{\lim _{\geq}} V_{\geq n} \hat{\otimes} W$. 
A $K S$-complex is a CDGA (commutative DGA) $(\bigwedge X, d)$, where $\bigwedge X=$ Exterior $\left(X^{\text {odd }}\right) \otimes \operatorname{Symmetric}\left(X^{\text {even }}\right)$ is the free CGA over the vector space $X=$ $X \geq 0$ which has a well-ordered basis $\left\{x_{\alpha}\right\}$ satisfying $d x_{\alpha} \in \Lambda\left(X_{<\alpha}\right)$. If $\left|x_{\alpha}\right|<$ $\left|x_{\beta}\right|$ implies $\alpha<\beta,(\bigwedge X, d)$ is a minimal KS-complex. A 1 -connected $\left(X^{0}=\right.$ $\left.X^{1}=0\right) \mathrm{KS}$-complex $(\bigwedge X, d)$ is minimal if and only if $d X \subset \wedge^{+} X \cdot \Lambda^{+} X$.

A $K S$-extension (resp. minimal $K S$-extension) of a CDGA $A$, augmented by $\varepsilon$, is a sequence of DGA maps of the form $A \stackrel{\text { id } \leftrightarrow 1}{\rightarrow}(A \otimes \wedge X, d) \stackrel{\varepsilon \otimes \text { id }}{\rightarrow}(\bigwedge X, \bar{d})$ where $(\bigwedge X, \bar{d})$ is a KS-complex (resp. minimal KS-complex) and $d x_{\alpha} \in A \otimes$ $\bigwedge X_{<\alpha}$.

Given a topological space $S$, consider the CDGA $A(S)$ of the polynomial forms on $S$. Then, there exists a quism (morphism inducing homology isomorphism) $\gamma:(\bigwedge X, d) \stackrel{\simeq}{\rightrightarrows} A(S)$ from a minimal KS-complex, unique up to isomorphism. $(\bigwedge X, d)$ together with $\gamma$ is the minimal model of $S$. If $S$ is 1 -connected and $H^{*}(S ; \mathbb{Q})$ has finite type, the $\mathbb{Q}$-vector spaces $X$ and $\operatorname{Hom}_{\mathbb{Z}}\left(\pi_{*}(S), \mathbb{Q}\right)$ are naturally identified.

In any case $A(S)$ and $C^{*}(S ; \mathbb{K})$ are connected by a chain of quisms, so that $H(\bigwedge X, d)$ and $H^{*}(S ; \mathbb{K})$ are naturally identified.

On the other hand, given a fibration $F \rightarrow E \stackrel{\rho}{\rightarrow} B \quad F$ and $B$ is simply connected, and $H^{*}(F ; \mathbb{Q})$ or $H^{*}(B ; \mathbb{Q})$ has finite type, there is a commutative diagram of CDGAs,

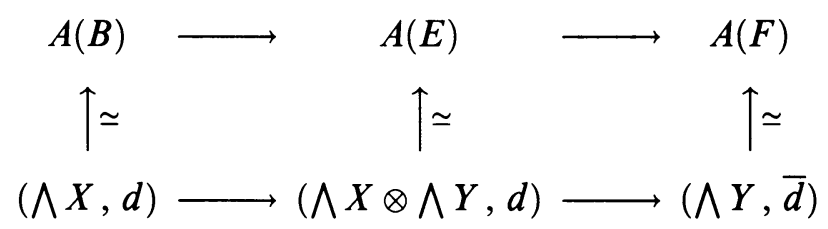

in which the bottom row is a KS-extension, $(\bigwedge X, d)$ and $(\bigwedge Y, \bar{d})$ are the minimal models of the base and the fibre respectively, and $(\bigwedge X \otimes \wedge Y, d)$ is the minimal model of the total space if and only if $\pi_{*}(\rho) \otimes \mathbb{Q}$ is surjective.

For notation and basic facts we shall use from differential homological algebra, we refer to [3 and 7].

Here, we shall simply recall some key notions:

Let $A=\left(A^{\#}, d\right)$ be a DGA. An $A$-module $M$ is a $A$-semifree if there exists a filtration of $A$-submodules $0=F_{-1} \subset F_{0} \subset F_{1} \subset \cdots$ such that $M=\bigcup_{i} F_{i}$ and for each $i, F_{i} / F_{i+1}$ is $A^{\#}$-free on a basis of cycles. A quism of $A$-modules $P \cong M$ is called a semifree resolution of $M$ if $P$ is semifree. Let $M, N$ be $A$-modules and $P \stackrel{\simeq}{\rightrightarrows} M$ a semifree resolution. Define $\operatorname{Ext}_{A}(M, N)=$ $H\left(\operatorname{Hom}_{A}(P, N)\right)$. This is independent of the choice of $P$.

Let $A$ be a DGA and let $M, N, W$ be $A$-modules in which $M$ is bounded below, i.e., $M=M^{\geq r}$, for some $r \in \mathbb{Z}$. Then define a vector map [7, Introduction],

$$
\psi: \operatorname{Ext}_{A}(M, N) \tilde{\otimes} \operatorname{Ext}_{A}(W, M) \rightarrow \operatorname{Ext}_{A}(W, N)
$$

as follows: Choose semifree resolutions $P \cong M$ and $Q \cong W$ of $M$ and $W$ respectively and let $\sum_{i \geq 0}\left[g_{i}\right] \otimes\left[f_{i}\right] \in \operatorname{Ext}_{A}(M, N) \tilde{\otimes} \operatorname{Ext}_{A}(W, M)$ in which $\left[g_{i}\right]$ 
and $\left[f_{i}\right]$ are represented by $g_{i}: P \rightarrow N$ and $f_{i}: Q \rightarrow M$. Then define

$$
\psi\left(\sum_{i \geq 0}\left[g_{i}\right] \otimes\left[f_{i}\right]\right)=\left[\sum_{i \geq 0} g_{i} \circ \bar{f}_{i}\right]
$$

where $\bar{f}_{i}: Q \rightarrow P$ is a homotopy lifting (see [3, Lemma A.3]) of $f_{i}$ to $P$ :

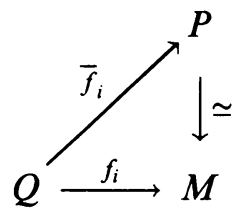

Note that $\psi$ is well defined (see [7] for details): Since $A$ is connected and $M$ is bounded below, $P$ can be chosen to be bounded below. Then, because $\operatorname{deg} f_{i} \rightarrow-\infty, \sum_{i} g_{i} \circ \bar{f}_{i}(x)$ is well defined for any $x \in Q$. Finally, it is straightforward to see that this map, $\sum_{i} g_{i} \circ \bar{f}_{i}$, is a cycle whose class depends only on $\left[g_{i}\right]$ and $\left[f_{i}\right]$.

Now, given a KS-extension $A \rightarrow(A \otimes \wedge Y, d) \rightarrow(\bigwedge Y, \bar{d})$ of the connected CDGA $A$, there is a natural map [7, Introduction]

$$
\varphi: \operatorname{Ext}_{A}(\mathbb{K}, A) \tilde{\otimes} \operatorname{Ext}_{\wedge Y}(\mathbb{K}, \bigwedge Y) \rightarrow \operatorname{Ext}_{A \otimes \wedge Y}(\mathbb{K}, A \otimes \wedge Y)
$$

defined as follows: The functor $-\otimes_{A}(A \otimes \Lambda Y)$ and the projection $(A \otimes$ $\bigwedge Y, d) \rightarrow(\bigwedge Y, \bar{d})$ induce natural maps

$$
\begin{aligned}
& \eta: \operatorname{Ext}_{A}(\mathbb{K}, A) \rightarrow \operatorname{Ext}_{A \otimes \wedge Y}(\bigwedge Y ; A \otimes \bigwedge Y), \\
& \nu: \operatorname{Ext}_{\wedge Y}(\mathbb{K}, \bigwedge Y) \rightarrow \operatorname{Ext}_{A \otimes \wedge Y}(\mathbb{K}, \bigwedge Y)
\end{aligned}
$$

Then define $\varphi=\psi \circ(\eta \tilde{\otimes} \nu)$.

1.1. Theorem [7, Theorems $\mathrm{A}$ and $\mathrm{B}]$. If either (i) $H^{*}(\bigwedge Y, \bar{d})$ is finite-dimensional or (ii) $(A \otimes \wedge Y, d)$ is the minimal KS-complex $(\bigwedge X \otimes \wedge Y, d)$ and $Y$ is finite-dimensional, then, ${ }^{1}$

$$
\varphi: \operatorname{Ext}_{A}(\mathbb{K}, A) \tilde{\otimes} \operatorname{Ext}_{\wedge Y}(\mathbb{K}, \bigwedge Y) \stackrel{\simeq}{\rightrightarrows} \operatorname{Ext}_{A \otimes \wedge Y}(\mathbb{K}, A \otimes \bigwedge Y)
$$

is an isomorphism.

\section{THE EVALUATION MAP}

Let $A$ be an augmented DGA. The evaluation map of $A$ is the natural linear $\operatorname{map}[3, \S 1]$,

$$
\mathrm{ev}_{A}: \mathrm{Ext}_{A}(\mathbb{K}, A) \rightarrow H(A),
$$

which assigns to each element $[f] \in \operatorname{Ext}_{A}(\mathbb{K}, A)$ represented by a cycle $f: P \rightarrow$ $A$ ( $P$ a semifree resolution of $\mathbb{K})$, the class $[f(p)] \in H(A)$ where $p$ is a cycle in $P$ representing 1 .

The evaluation map $\mathrm{ev}_{S}$ of a pointed topological space $S$ is the evaluation map of $C^{*}(S ; \mathbb{K})$ (or of any other DGA with the same weak homotopy type).

\footnotetext{
${ }^{1}$ In both cases the semicomplete tensor product coincides with the classical one.
} 
2.1. Remarks. (1) Let $A \cong B$ be a quism of augmented DGAs. Then it induces an isomorphism $\operatorname{Ext}_{A}(\mathbb{K}, A) \stackrel{\simeq}{\rightrightarrows} \operatorname{Ext}_{B}(\mathbb{K}, B)$. One can easily see that the following diagram commutes

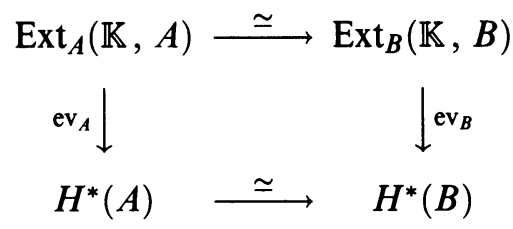

Therefore, we can use the quism $A \cong B$ to identify $\mathrm{ev}_{A} \equiv \mathrm{ev}_{B}$. Then, for any pointed space $S, \mathrm{ev}_{S}=\operatorname{ev}_{C^{*}(S ; \mathbf{K})}=\operatorname{ev}_{A(S)}=\operatorname{ev}_{\wedge X}$, with $(\bigwedge X, d)$ the minimal model of $S$.

(2) Given a $c$-connected DGA $A\left(H^{0}(A)=\mathbb{K}\right), " \mathrm{ev}_{A} \neq 0$ " is an intermediate property between "having a cohomology class represented by an element which annihilates $A^{+}$" and "having a cohomology class which annihilates $H^{+}(A)$."

In fact, if $a \in A$ is a cycle different from zero and such that $a \cdot A^{+}=0$, by [3, Lemma 1.5], $[a] \in \operatorname{Imev}_{A}$. On the other hand, if $\operatorname{ev}_{A} \neq 0$, choose $\alpha \in \operatorname{Imev}_{A}, \alpha \neq 0$. Hence, $\alpha=H(f)[p]=[f(p)]$ for some $p \in P, P$ a semifree resolution of $\mathbb{K}$, and $f: P \rightarrow A$, homomorphism of $A$-modules. Then, for any $\beta=[\Phi] \in H^{+}(A)$,

$$
\beta \cdot \alpha=[\Phi f(p)]=(-1)^{|f||\Phi|}[f(\Phi \cdot p)]=(-1)^{|f||\Phi|} H(f)[\Phi \cdot p]=0,
$$

since $H^{+}(A) \cdot H(P)=H^{+}(A) \cdot \mathbb{K}=0$.

(3) Moreover, if $A$ is a $c$-connected DGA and $H^{>n}(A)=0$ for some $n \in \mathbb{Z}$, then $H^{n}(A) \subset \operatorname{Imev}_{A}$. In fact (we assume $A$ connected since we can always replace $A$ by an equivalent connected DGA), consider the quotient DGA $A / I$ in which $I=A^{>n} \oplus P$ with $P \subset A^{n}$ the complement of the cocycles in degree $n$. $I$ is acyclic so the projection $A \cong A$ 年 $A / I$ is a quism and since $(A / I)^{>n}=0$, $H^{n}(A) \subset \operatorname{Im~ev}_{A}$.

Now we shall give two other equivalent descriptions of the evaluation map:

First approach. Let $M$ and $N$ be $A$-modules. A linear map, natural in all possible ways,

$$
\operatorname{ev}(A ; M, N): \operatorname{Ext}_{A}(M, n) \rightarrow \operatorname{Hom}_{H(A)}(H(M), H(N)),
$$

is defined as follows: Let $[g] \in \operatorname{Ext}_{A}(M, N)$ be represented by $g: P \rightarrow N$, with $P \cong$ an $A$-semifree resolution of $M$, and let $\alpha$ be an element of $H(M)$. Define $\operatorname{ev}(A ; M, N)[g](\alpha)=[g(p)]$, with $p \in P$ a cycle representing $\alpha$.

For $M=\mathbb{K}, N=A$, we get a map

$$
\operatorname{ev}(A ; \mathbb{K}, A): \operatorname{Ext}_{A}(\mathbb{K}, A) \rightarrow \operatorname{Hom}_{H(A)}(\mathbb{K}, H(A)),
$$

which composed with the morphism $\operatorname{Hom}_{H(A)}(\mathbb{K}, H(A)) \rightarrow \operatorname{Hom}_{\mathbb{K}}(\mathbb{K}, H(A)) \cong$ $H(A)$, induced by the inclusion $\mathbb{K} \hookrightarrow H(A)$, gives $\mathrm{ev}_{A}$.

2.2. Remark. Given $M, N, W A$-modules, with $M$ bounded below, it is a straightforward computation to show that the following diagram, where $\alpha$ is 
just the composition, commutes:

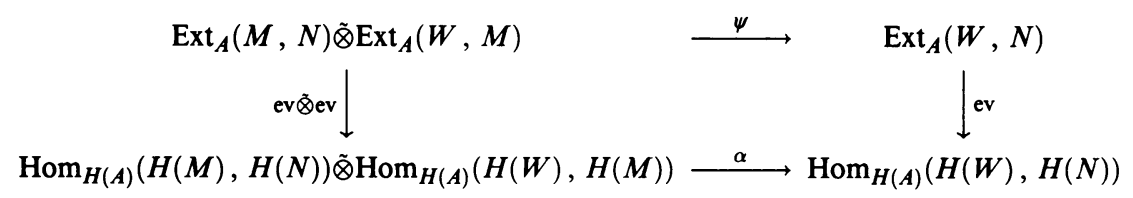

Second approach. Recall that a proper projective resolution of an $A$-module $M[8, \S 1]$ is a sequence of $A$-module morphism of degree 0 ,

$$
\cdots \rightarrow M(n-1) \stackrel{d}{\rightarrow} M(n) \rightarrow \cdots \stackrel{d}{\rightarrow} M(0) \rightarrow M \rightarrow 0
$$

(indexed by the nonpositive integers) such that

(i) the sequences

$$
\begin{gathered}
\ldots \rightarrow M(n-1) \stackrel{d}{\rightarrow} M(n) \rightarrow \cdots \stackrel{d}{\rightarrow} M(0) \rightarrow M \rightarrow 0, \\
\cdots \rightarrow Z(M(n-1)) \rightarrow Z(M(n)) \rightarrow \cdots \rightarrow Z(M(0)) \rightarrow Z(M) \rightarrow 0, \\
\cdots \rightarrow H(M(n-1)) \rightarrow H(M(n)) \rightarrow \cdots \rightarrow H(M(0)) \rightarrow H(M) \rightarrow 0
\end{gathered}
$$

are exact sequences of vector spaces $(Z(M(n))$ denotes the space of cycles in $M(n))$.

(ii) The modules $M(i), i \geq 0$, are proper projective, that is to say, given a surjective homomorphism of $A$-modules $f: P \rightarrow Q$ where the map $Z(f)$ : $Z(P) \rightarrow Z(Q)$ is surjective, and $g: M(i) \rightarrow Q$, there exists a homomorphism $h$ :

$M(i) \rightarrow P$ such that $h \circ f=g$.

Given such a proper projective resolution, the sequence

$$
\cdots \rightarrow H(M(n-1)) \rightarrow H(M(n)) \rightarrow \cdots \rightarrow H(M(0)) \rightarrow H(M) \rightarrow 0
$$

is a projective resolution of $H(M)$ as $H(A)$-modules [8, $\S 1]$.

Observe that we can regard a proper projective resolution of $M$ as an $A$ module $(P, D)$ where $P^{r}=\bigoplus_{n+q=r} M^{q}(n)$ and $D(\Phi)=(-1)^{n} d(\Phi)+d_{I}(\Phi)$, $\Phi \in M(n)$, with $d_{I}$ the internal differential in each $M(n)$.

Given an $A$-module $N$, filter $\operatorname{Hom}_{A}(P, N)$ by $F^{j}=\left\{f \in \operatorname{Hom}_{A}(P, N)\right.$, $f(M(<j))=0\}$. It is easy to see from (1) that the $E_{2}$-term of the resulting spectral sequence is

$$
E_{2}=\operatorname{Ext}_{H(A)}(H(M), H(N))
$$

In particular,

$$
E_{2}^{0, *}=\operatorname{Hom}_{H(A)}(H(M), H(N)) .
$$

On the other hand, observe that

$$
E_{i}^{0, *} \subset E_{i-1}^{0, *} \subset \cdots \subset E_{2}^{0, *} .
$$

Now, since $P \stackrel{\cong}{\rightrightarrows} M$ is a quism [8, Lemma 3.3], $\operatorname{Ext}_{A}(M, N) \stackrel{\cong}{\models} \operatorname{Ext}_{A}(P, N)$ [7, Remark 1.6(2)] or [3, Proposition A.4]. Then the $E_{\infty}$-term is, by definition, the associated graded space of $\operatorname{Ext}_{A}(M, N)$ :

$$
\left[\operatorname{Ext}_{A}(M, N)\right]^{n} / F^{1, n-1} \simeq E_{\infty}^{0, n} .
$$


$\left(F^{i, j}\right.$ denotes here the filtration induced in $\operatorname{Ext}_{A}(M, N)$.) Although this spectral sequence may not be convergent, observe that there are morphisms $E_{\infty}^{0, q} \rightarrow$ $E_{i}^{0, q}$ which make the following diagram commutative for any $i$ :

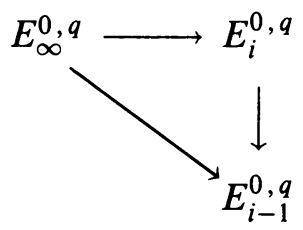

Combining this fact together with (2), (3), and (4), we get a map

$$
\operatorname{Ext}_{A}(M, N) \rightarrow \operatorname{Hom}_{H(A)}(H(M), H(N)) \text {. }
$$

It is then easy to check that this map coincides with $\operatorname{ev}(A ; M, N)$.

\section{INTEGRATION OVER THE FIBRE AND}

A CHARACTERIZATION OF ELLIPTIC SPACES

In view of the connection between Sullivan's theory of minimal models and rational homotopy theory, Theorem B(i) will be established once we prove

3.1. Theorem. Let $(A \otimes \wedge Y, d)$ be a KS-extension of the 1-connected CDGA $A$ in which $H^{*}(\Lambda Y, \bar{d})$ is finite-dimensional. Then $\mathrm{ev}_{A} \neq 0$ implies $\mathrm{ev}_{A \otimes \wedge Y} \neq 0$.

In the same way, Theorem $\mathrm{A}$ is deduced from

3.2. Theorem. Let $(\bigwedge X, d)$ be a 1-connected minimal KS-complex where the vector space $X$ is finite-dimensional. Then

$$
\operatorname{dim} H^{*}(\bigwedge X, d)<\infty \text { if and only if } \operatorname{ev}_{(\wedge X, d)} \neq 0 .
$$

The rest of this section is devoted to the proof of those two results.

Let $A \rightarrow(A \otimes \wedge Y, d) \rightarrow(\bigwedge Y, \bar{d})$ be a KS-extension of the 1-connected CDGA $A$, and filter $A \otimes \wedge Y$ by $F^{p}=A^{\geq p} \otimes \wedge Y$. The resulting Serre spectral sequence is a first quadrant spectral sequence, and hence convergent. Its $E_{2}$ term is of the form $E_{2}^{p, q}=H^{p}(A) \otimes H^{q}(\bigwedge Y)$.

Suppose now that there exists an integer $N$ such that $H^{q}(\bigwedge Y)=0, q>N$. Then, since $E_{1}^{p, q}=0, q>N$, we have

$$
E_{\infty}^{*, N} \subset \cdots \subset E_{i}^{*, N} \subset E_{i-1}^{*, N} \subset \cdots \subset E_{2}^{*, N}=H^{*}(A) \otimes H^{N}(\bigwedge Y) .
$$

On the other hand, if $F(H)$ denotes the induced filtration in $H^{*}(A \otimes \wedge Y)$, the convergence of the spectral sequence means in particular that

$$
H^{p}(A \otimes \bigwedge Y) / F^{p-N+1, N-1}(H)=E_{\infty}^{p-N, N}, \quad p \geq 0 .
$$

Composing (5) and (6) we get a linear map

$$
\int_{\Lambda Y}: H^{*}(A \otimes \bigwedge Y) \rightarrow E_{\infty}^{*-N, N} \hookrightarrow H^{*}(A) \otimes H^{N}(\bigwedge Y) ;
$$

it expresses the integration over the fibre (defined by Spanier in [9]. See also [4, Chapter VII, §5]) in terms of models.

Conversely, if we assume that $M$ is the largest integer for which $H^{M}(A) \neq 0$ we get from the Serre spectral sequence:

$$
H^{M}(A) \otimes H(\bigwedge Y)=E_{2}^{M, *} \rightarrow E_{3}^{M, *} \rightarrow \cdots \rightarrow E_{\infty}^{M, *} .
$$


On the other hand,

$$
E_{\infty}^{M, *}=F^{M}(H(A \otimes \bigwedge Y)) \hookrightarrow H(A \otimes \bigwedge Y)
$$

Hence, composing (7) and (8) we obtain a linear map

$$
\beta: H^{M}(A) \otimes H(\bigwedge Y) \rightarrow H(A \otimes \bigwedge Y)
$$

Our first step in proving Theorems 3.1 and 3.2 is to establish the following results.

3.3. Theorem. Let $(A \otimes \bigwedge Y, d)$ be a KS-extension in which $H^{*}(\bigwedge, \bar{d})$ is finite-dimensional. Let $N$ be the largest integer for which $H^{N}(\bigwedge Y, \bar{d}) \neq 0$. Then the following diagram commutes:

$$
\begin{aligned}
& \operatorname{Ext}_{A}(\mathbb{K}, A) \otimes \operatorname{Ext}_{\wedge Y}^{N}(\mathbb{K} ; \bigwedge Y) \stackrel{\varphi}{\longrightarrow} \operatorname{Ext}_{A \otimes \wedge Y}(\mathbb{K}, A \otimes \wedge Y) \\
& \mathrm{ev}_{A} \otimes \mathrm{ev} \wedge Y \downarrow \quad \downarrow \mathrm{ev}_{A \otimes} \wedge_{Y} \\
& H^{*}(A) \otimes H^{N}(\bigwedge Y) \quad \stackrel{\int_{\bigwedge_{Y}}}{\longleftarrow} \quad H^{*}(A \otimes \bigwedge Y)
\end{aligned}
$$

Remark. In Theorem 3.3, $\varphi$ is the restriction to $\operatorname{Ext}_{A}(\mathbb{K}, A) \otimes \operatorname{Ext}_{\wedge Y}^{N}(\mathbb{K} ; \bigwedge Y)$ of the isomorphism

$$
\varphi: \operatorname{Ext}_{A}(\mathbb{K}, A) \tilde{\otimes} \operatorname{Ext}_{\wedge Y}(\mathbb{K}, \bigwedge Y) \stackrel{\cong}{\rightarrow} \operatorname{Ext}_{A \otimes \wedge Y}(\mathbb{K}, A \otimes \bigwedge Y),
$$

given in $\S 1$.

Proof. First, we shall give an explicit formula for the isomorphism $\varphi$ in this particular case. Consider a Sullivan model $(A \otimes \wedge X, d) \cong \mathbb{\rightrightarrows}$; it is an $A$ semifree resolution of $\mathbb{K}$. In the same way, $(A \otimes \wedge X, d) \otimes_{A}(A \otimes \Lambda Y, d)=$ $(A \otimes \wedge X \otimes \wedge Y, d)$ is an $A \otimes \wedge Y$-semifree resolution of $(\bigwedge Y, \bar{d})$ via the quism given by the projection

$$
(A \otimes \bigwedge X \otimes \bigwedge Y, d) \stackrel{\cong}{\rightrightarrows}(A \otimes \bigwedge X \otimes \bigwedge Y, d) /(A \otimes \bigwedge X)^{+} \otimes \bigwedge Y=(\bigwedge Y, \bar{d}) .
$$

On the other hand a model $(A \otimes \wedge X \otimes \bigwedge Y \otimes \bigwedge \bar{Y}, d)$ of the projection $(A \otimes$ $\bigwedge X \otimes \wedge Y, d) \rightarrow \mathbb{K}$ is an $(A \otimes \wedge Y, d)$-semifree resolution of $\mathbb{K}$. Dividing by the ideal generated by $(A \otimes \wedge X)^{+}$we get the $\wedge Y$-semifree resolution of $\mathbb{K}:(\bigwedge Y \otimes \bigwedge \bar{Y}, \bar{d}) \stackrel{\simeq}{\rightrightarrows} \mathbb{K}$.

Let $[f] \in \operatorname{Ext}_{\wedge Y}(\mathbb{K}, \wedge Y)$ and $[g] \in \operatorname{Ext}_{A}(\mathbb{K}, A)$ be represented by $g:(A \otimes$ $\bigwedge X) \rightarrow A$ and $f:(\bigwedge Y \otimes \bigwedge \bar{Y}, \bar{d}) \rightarrow(\bigwedge Y, \bar{d})$. Then $\eta[g]$ is represented by $\tilde{g} \in \operatorname{Hom}_{A \otimes \wedge Y}(A \otimes \wedge X \otimes \wedge Y, A \otimes \wedge Y), \tilde{g}=g \otimes 1_{\wedge Y}$. On the other hand, there is an $A \otimes \wedge X \otimes \wedge Y$-linear map, $\tilde{f}:(A \otimes \wedge X \otimes \wedge Y \otimes \wedge \bar{Y}, d) \rightarrow$ $(A \otimes \wedge X \otimes \wedge Y, d)$ such that the following diagram commutes:

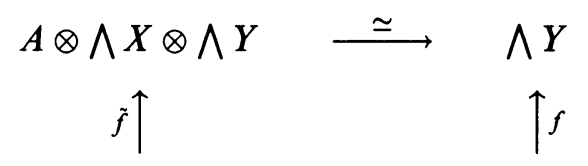


By definition $\nu[f]$ is represented by $\tilde{f}$ and hence, $\varphi([g] \otimes[f])=[\tilde{g} \circ \tilde{f}]$. Choose $[f] \in \operatorname{Ext}_{\wedge Y}^{N}(\mathbb{K}, \bigwedge Y)$. In view of diagram (9),

$$
\tilde{f}(1)=1 \otimes f(1)-\Omega, \quad \Omega \in(A \otimes \bigwedge X)^{+} \otimes \bigwedge Y, \quad d(1 \otimes f(1))=d \Omega .
$$

Write $\Omega=\sum_{i} \alpha_{i} \beta_{i}$, with $\alpha_{i} \in(A \otimes \bigwedge X)^{+}$and $\beta_{i} \in(\bigwedge Y)^{<N}$. In view of the definition of the evaluation map given at the start of $\S 2$ we obtain

$$
\left(\mathrm{ev}_{A \otimes \wedge Y} \circ \varphi\right)([g] \otimes[f])=[(\tilde{g} \circ \tilde{f})(1)]=\left[g(1) \otimes f(1)-\sum_{i} g\left(\alpha_{i}\right) \otimes \beta_{i}\right] .
$$

Finally, observe that

$$
\int_{\wedge Y}\left[g(1) \otimes f(1)-\sum_{i} g\left(\alpha_{i}\right) \otimes \beta_{i}\right]=[g(1)] \otimes[f(1)]=\operatorname{ev}_{A}[g] \otimes \operatorname{ev}_{\wedge Y}[f] .
$$

3.4. Theorem. Let $(A \otimes \bigwedge Y, d)$ be a KS-extension in which $A$ is 1-connected and $H(A)$ is finite-dimensional (let $M$ be the largest integer for which $H^{M}(A) \neq$ $0)$. Then the following diagram commutes:

$$
\begin{aligned}
& \operatorname{Ext}_{A}^{M}(\mathbb{K}, A) \otimes \operatorname{Ext}_{\wedge Y}(\mathbb{K} ; \bigwedge Y) \stackrel{\varphi}{\rightarrow} \operatorname{Ext}_{A \otimes \wedge Y}(\mathbb{K}, A \otimes \wedge Y) \\
& \downarrow \mathrm{ev}_{A} \otimes \mathrm{ev} \wedge_{Y} \\
& H^{M}(A) \otimes H^{*}(\bigwedge Y) \\
& \stackrel{\beta}{\rightarrow} \quad H^{*}(A \otimes \bigwedge Y)
\end{aligned}
$$

Proof. Straightforward using the same explicit formula of $\varphi$ given in the proof of Theorem 3.3.

Proof of Theorem 3.1. Let $N$ be the largest integer such that $H^{N}(\bigwedge Y) \neq 0$. By Remark 2.1(3) $H^{N}(\bigwedge Y, \bar{d}) \subset \operatorname{Imev} \wedge Y$. Apply Theorem 3.3 to conclude that $\mathrm{ev}_{A} \neq 0$ implies $\mathrm{ev}_{A \otimes \wedge Y} \neq 0$.

3.5. Proposition. Let $(\bigwedge X, d)$ be a minimal $K S$-complex in which $X$ is finitedimensional. Write $\bigwedge X=\bigwedge\left(x_{1}, \ldots, x_{n}\right)$. Then, for any $i, \operatorname{ev} \wedge\left(x_{1}, \ldots, x_{n}\right)$ $\neq 0$ implies $\operatorname{ev} \wedge\left(x_{i}, \ldots, x_{n}\right) \neq 0$.

Proof. We shall prove that $\operatorname{ev} \wedge\left(x_{2}, \ldots, x_{n}\right) \neq 0$ and an obvious induction will complete the proof.

Suppose first that $\left|x_{1}\right|$ is even. Then define a KS-extension, $\left(\bigwedge\left(x_{1}, \ldots, x_{n}\right) \otimes\right.$ $\bigwedge y, d), d y=x_{1}$, whose minimal model is $\left(\bigwedge\left(x_{2}, \ldots, x_{n}\right), \bar{d}\right)$ (see [5, Chapter 2]). Next, $H^{*}(\bigwedge y, 0)=\bigwedge y$ is finite-dimensional ( $y$ has odd degree). Thus, by Theorem 3.1, ev $\wedge\left(x_{1}, \ldots, x_{n}\right) \otimes \wedge y \neq 0$, or equivalently, $\operatorname{ev} \wedge\left(x_{2}, \ldots, x_{n}\right) \neq 0$.

Assume now that $\left|x_{1}\right|$ is odd. By Theorem 1.1,

$$
\begin{aligned}
\varphi: \operatorname{Ext}_{\wedge x_{1}}\left(\mathbb{K}, \bigwedge x_{1}\right) & \otimes \operatorname{Ext}_{\wedge x_{2}, \ldots, x_{n}}\left(\mathbb{K}, \bigwedge x_{2}, \ldots, x_{n}\right) \\
& \cong \operatorname{Ext}_{\lambda x_{1}, \ldots, x_{n}}\left(\mathbb{K}, \bigwedge x_{1}, \ldots, x_{n}\right),
\end{aligned}
$$

is an isomorphism. Since $H\left(\bigwedge x_{1}, 0\right)$ is finite-dimensional, we may apply $\mathrm{Re}$ mark 2.1(3) and Theorem 3.4 to obtain that $\operatorname{ev} \wedge\left(x_{2}, \ldots, x_{n}\right) \neq 0$.

Proof of Theorem 3.2. (a) Suppose that $H^{*}(\bigwedge X, d)$ is finite-dimensional and $N$ is such that $H^{N}(\bigwedge X, d) \neq 0$ and $H^{p}(\bigwedge X, d)=0, p>N$. By Remark 2.1(3), $H^{N}(\bigwedge X, d) \subset \operatorname{Imev} \wedge X$.

(b) Suppose that $e_{\wedge x} \neq 0$. We shall proceed by induction on $\operatorname{dim} X$. Assume $X=(x)$ of dimension 1. If $x$ has even degree, $\operatorname{Ext}_{\wedge x}(\mathbb{K}, \wedge x)$ is 
generated by $[g]$, with $g:(\bigwedge(x, \bar{x}), d) \rightarrow(\bigwedge x, 0), d \bar{x}=x, g(1)=0$, and $g(\bar{x})=1$, which contradicts the fact $\operatorname{ev} \wedge x \neq 0$. Therefore $|x|$ is odd and then $H^{*}(\bigwedge x, 0)$ is finite-dimensional.

Suppose our assertion is true for $\operatorname{dim} X \leq n-1$.

Write $X=\left(x_{1}, \ldots, x_{n}\right)$. By Proposition 3.5, $\operatorname{ev}_{\wedge\left(x_{2}, \ldots, x_{n}\right)} \neq 0$. Thus, $H^{*}\left(\bigwedge\left(x_{2}, \ldots, x_{n}\right), \bar{d}\right)$ is finite-dimensional.

If $\left|x_{1}\right|$ is odd, it is clear that $H^{*}(\bigwedge X, d)$ is also finite-dimensional. Suppose $x_{1}$ of degree even. We shall find a contradiction assuming

$$
\operatorname{ev}_{\wedge X} \neq 0 \text { and } \operatorname{dim} H^{*}(\bigwedge X, d)=\infty
$$

For this we rely on another characterization of rationally elliptic spaces $[6$, Proposition 5.1] according to which a minimal KS-complex $(\bigwedge X, d)$ (defined over an algebraically closed field $\overline{\mathbb{K}})$, with $X$ finite-dimensional, has finitedimensional homology if and only if the only morphism $(\bigwedge X, d) \rightarrow(\bigwedge a, 0)$, $|a|=2$, is the trivial one. To apply this result we reduce to the case $\mathbb{K}$ is algebraically closed: Let $\overline{\mathbb{K}}$ be the algebraic closure of $\mathbb{K}$. Then for any KScomplex $(\bigwedge X, d)$ we have the commutative diagram

$$
\begin{aligned}
& \operatorname{Ext}_{\wedge X \otimes_{\mathbf{K}} \overline{\mathbf{K}}}\left(\overline{\mathbb{K}}, \wedge X \otimes_{\mathbf{K}} \overline{\mathbb{K}}\right) \hookleftarrow \operatorname{Ext}_{\wedge X}(\mathbb{K}, \wedge X) \\
& \operatorname{lev} \wedge x \otimes_{\mathbf{K}} \overline{\mathbf{R}} \quad \operatorname{lev} \wedge x \\
& H\left(\bigwedge X \otimes_{\mathbb{K}} \overline{\mathbb{K}}\right) \quad \hookleftarrow \quad H(\bigwedge X)
\end{aligned}
$$

Thus, $\mathrm{ev}_{\wedge X \otimes_{\mathbf{K}} \overline{\mathbf{K}}} \neq 0$ and $\operatorname{dim} H^{*}\left(\bigwedge X \otimes_{\mathbf{K}} \overline{\mathbb{K}}\right)=\infty$. It is therefore sufficient to show that (10) leads to a contradiction when $\mathbb{K}$ is algebraically closed and we assume this henceforth. Apply then [6, Proposition 5.1] to get a nontrivial homomorphism $\left(\bigwedge\left(x_{1}, \ldots, x_{n}\right), d\right) \stackrel{p}{\rightarrow}(\mathbb{K}[a], 0)$. Moreover, we can assume $\gamma\left(x_{1}\right)=\lambda a^{p} \neq 0$. Otherwise, $\gamma$ factors over $\left(\bigwedge\left(x_{1}, \ldots, x_{n}\right), d\right) \rightarrow$ $\left(\bigwedge\left(x_{1}, \ldots, x_{n}\right) /\left(x_{1}\right), \bar{d}\right) \cong\left(\bigwedge\left(x_{2}, \ldots, x_{n}\right), \bar{d}\right)$,

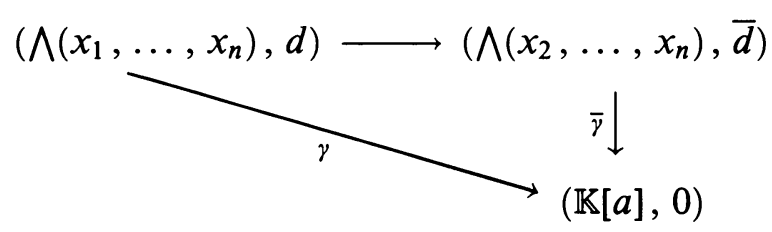

and thus $\bar{\gamma}$ is a nontrivial morphism, which contradicts the fact that

$$
H^{*}\left(\bigwedge\left(x_{2}, \ldots, x_{n}\right)\right)
$$

is finite-dimensional. Observe that since $\gamma\left(x_{1}\right) \neq 0, d x_{2}=0$. Now, define a KS-extension of $\left(\Lambda\left(x_{1}, \ldots, x_{n}\right), d\right)$,

$$
\left(\bigwedge\left(x_{1}, \ldots, x_{n}\right) \otimes \bigwedge(a, y), d\right), \quad d a=0, \quad d y=x_{1}-\lambda a^{p},
$$

whose minimal model has the form $\left(\Lambda\left(a, x_{2}, \ldots, x_{n}\right), d\right)$. Since

$$
H^{*}(\bigwedge(a, y), \bar{d})
$$

is finite-dimensional, by Theorem 3.1, $\operatorname{ev}_{\wedge\left(a, x_{2}, \ldots, x_{n}\right)} \neq 0$. Since $d x_{2}=0$, $\left(\bigwedge\left(a, x_{2}, \ldots, x_{n}\right), d\right)=\left(\bigwedge\left(x_{2}, a, \ldots, x_{n}\right), d\right)$. Hence, by the induction hypothesis and Proposition 3.5, $\operatorname{dim} H^{*}\left(\Lambda\left(a, x_{3}, \ldots, x_{n}\right)\right)<\infty$. 
On the other hand, $\gamma$ extends to $\left(\bigwedge\left(x_{1}, \ldots, x_{n}\right) \otimes \bigwedge(a, y), d\right)$ by $\gamma(a)=$ $a$ and $\gamma(y)=0$, so it induces a morphism $\gamma: P\left(\Lambda\left(a, x_{2}, \ldots, x_{n}\right), d\right) \rightarrow$ $(\mathbb{K}[a], 0), \gamma(a)=a$. If $\gamma\left(x_{2}\right)=\lambda a^{q}$ replace $x_{2}$ by $x_{2}-\lambda a^{q}$ in $\Lambda\left(a, x_{2}, \ldots, x_{n}\right)$ so that we can assume $\gamma\left(x_{2}\right)=0$. Therefore, $\gamma$ factors over the ideal generated by $x_{2}$ to give a nontrivial homomorphism $\bar{\gamma}:\left(\bigwedge\left(a, x_{3}, \ldots, x_{n}\right), \bar{d}\right) \rightarrow$ $(\mathbb{K}[a], 0)$. But, again by [6, Proposition 5.1], this is a contradiction to the fact that $H^{*}\left(\bigwedge\left(a, x_{3}, \ldots, x_{n}\right)\right)$ is finite-dimensional.

\section{A COMMUTATIVE DiAgRAM}

Theorem B(ii) will be established once we prove the following.

4.1. Theorem. Let $(\bigwedge X, d) \rightarrow(\bigwedge X \otimes \bigwedge Y, d) \rightarrow(\bigwedge Y, \bar{d})$ be a minimal $K S$-extension of the 1-connected minimal KS-complex $(\Lambda X, d)$ in which the $K S$-complex $(\bigwedge X \otimes \bigwedge Y, d)$ is also minimal and $Y$ is finite-dimensional. Then ev $\wedge X \otimes \wedge Y \neq 0$ implies ev $\wedge Y \neq 0$.

Proof of Theorem 4.1. Consider the diagram of Remark 2.2 which in this particular setting reads

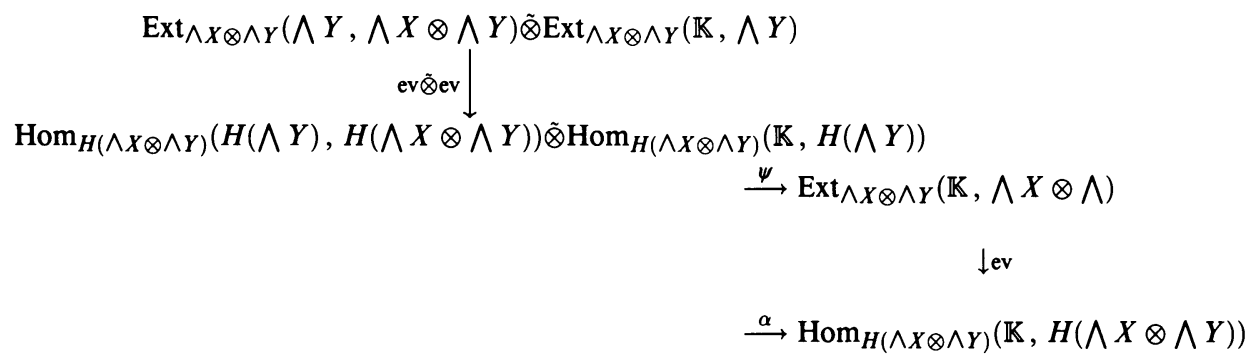

By Theorem 1.1,

$$
\varphi: \operatorname{Ext}_{\wedge X}(\mathbb{K}, \bigwedge X) \otimes \operatorname{Ext}_{\wedge Y}(\mathbb{K}, \bigwedge Y) \stackrel{\cong}{\rightarrow} \operatorname{Ext}_{\wedge X \otimes \wedge Y} \operatorname{Ext}_{\wedge X \otimes \wedge}(\mathbb{K}, \bigwedge X \otimes \bigwedge Y)
$$

is an isomorphism, because the hypothesis $\operatorname{dim} Y<\infty \operatorname{implies} \operatorname{Ext}_{\wedge Y}(\mathbb{K}, \bigwedge Y)$ is one-dimensional. Thus, in the diagram above $\psi$ is surjective. Since $e v \wedge x \otimes \wedge Y$ $\neq 0$, it follows that $\operatorname{ev}(\bigwedge X \otimes \wedge Y ; \mathbb{K}, \bigwedge Y) \neq 0$. Thus, by the communtativity of the diagram

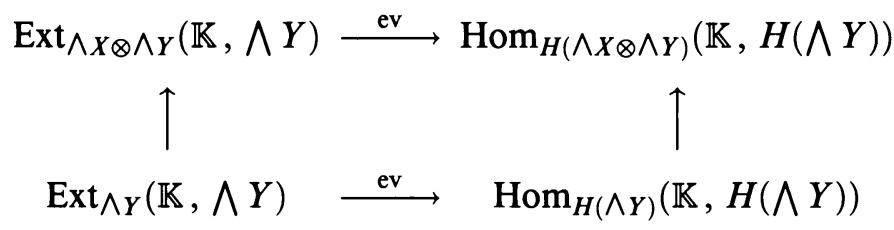

$\operatorname{ev}(\bigwedge Y ; \mathbb{K}, \bigwedge Y) \neq 0$. Therefore, since the map $\operatorname{Hom}_{H(\wedge Y)}(\mathbb{K}, H(\bigwedge Y)) \rightarrow$ $\operatorname{Hom}_{\mathbf{K}}(\mathbb{K}, H(\bigwedge Y)) \cong H(\bigwedge Y)$ is injective, it follows that $\operatorname{ev}_{\wedge} Y \neq 0$.

\section{ACKNOWLEDGMENTS}

This paper is based on part of my thesis, and I am indebted to my supervisor, Professor Stephen Halperin of the University of Toronto, for his patience, help, and encouragement. Also I would like to thank the referee for his careful reading of the preprint and his helpful comments, especially in the proof of Theorem 3.4. 


\section{REFERENCES}

1. Y. Félix and S. Halperin, Rational L-S category and its applications, Trans. Amer. Math. Soc. 273 (1982), 1-37.

2. Y. Félix, S. Halperin, J. M. Lemairè, and J. C. Thomas, Mod ploop space homology, Invent. Math. 92 (1989), 247-262.

3. Y. Félix, S. Halperin, and J. C. Thomas, Gorenstein spaces, Adv. in Math. 71 (1988), 92112.

4. W. Greub, S. Halperin, and R. Vanstone, Connections, curvature, and cohomology, vol. III, Academic Press, New York, 1972.

5. S. Halperin, Lectures on minimal models, Mém. Soc. Math. France 9/10 (1983).

6. __ Torsion gaps for finite complexes. II, Topology 30 (1991), 471-478.

7. A. Murillo, Rational fibrations in differential homological algebra, Trans. Amer. Math. Soc. 332 (1992), 79-91.

8. L. Smith, Homological algebra and the Eilenberg-Moore spectral sequence, Trans. Amer. Math. Soc. 129 (1970), 58-93.

9. E. H. Spanier, Homology theory of fiber bundles, Proc. Internat. Congr. Math., Cambridge, Mass., 1950, pp. 390-396.

10. D. Sullivan, Infinitesimal computations in topology, Inst. Hautes Études Sci. Publ. Math. 47 (1978), 269-331.

Departamento de Algebra Geometría y Topología, Universidad de Málaga, Ap. 59, 29080 Málaga, Spain

E-mail address: aniceto@ccuma.uma.es 